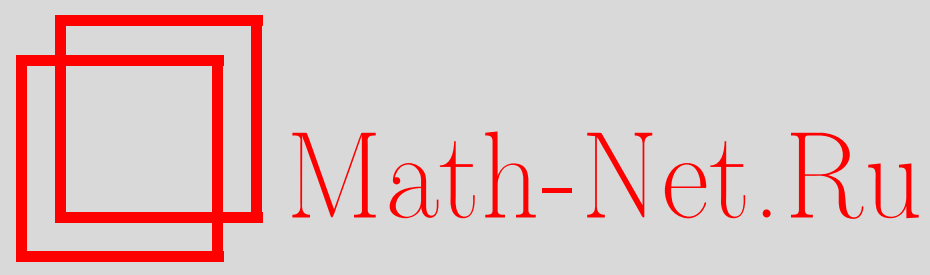

Е. И. Галахов, Положительные решения квазилинейного эллиптического уравнения, Матем. заметки, 2005, том 78, выпуск 2, 202-211

DOI: https://doi.org/10.4213/mzm2577

Использование Общероссийского математического портала Math-Net.Ru подразумевает, что вы прочитали и согласны с пользовательским соглашением http://www . mathnet.ru/rus/agreement

Параметры загрузки:

IP : 54.166.219.16

26 апреля 2023 г., 18:31:22

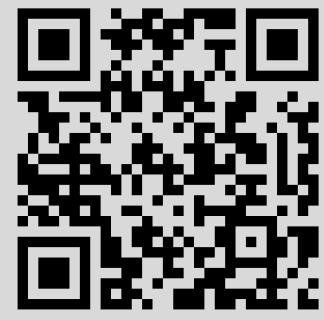




\section{ПОЛОЖИТЕЛЬНЫЕ РЕШЕНИЯ КВАЗИЛИНЕЙНОГО ЭЛЛИПТИЧЕСКОГО УРАВНЕНИЯ}

\section{Е.И. Галахов}

Работа посвящена теоремам существования положительных решений задачи Дирихле для квазилинейного эллиптического дифференциального уравнения, содержащего градиентный член. Методом стрельбы с помощью априорных оценок для первого нуля получены достаточные условия существования классических положительных решений задачи в шаре.

Библиография: 4 названия.

1. Введение. Рассмотрим задачу

$$
\begin{cases}-\Delta_{p} \varphi(x)=\lambda \varphi^{q}(x)-|\nabla \varphi(x)|^{s}, & x \in B_{R}(0) \\ \varphi(x)>0, & x \in B_{R}(0) \\ \varphi(x)=0, & x \in \partial B_{R}(0)\end{cases}
$$

где $\Delta_{p} \varphi:=\operatorname{div}\left(|\nabla \varphi|^{p-2} \nabla \varphi\right), p>1, q \geqslant 1, s \geqslant p-1, \lambda>0$, a $B_{R}(0)=\left\{x \in \mathbb{R}^{n}:|x|<R\right\}$. В настоящей статье мы ограничимся поиском решений в классе радиально-симметричных функций. Мотивировкой для этого могут послужить известные результаты о радиальной симметрии решений задачи (1.1) в случае $p=2$ [1] и аналогичной задачи с произвольным $p>1$, но без градиентного члена [2]. В этом классе решения (1.1) можно искать, в частности, в виде функций $\varphi(x):=u(|x|)$, где $u(r)$ - положительное решение вспомогательной начальной задачи

$$
\left\{\begin{array}{l}
-\left((p-1) u^{\prime \prime}(r)+\frac{n-1}{r} u^{\prime}(r)\right)\left|u^{\prime}(r)\right|^{p-2}=\lambda u^{q}(r)-\left|u^{\prime}(r)\right|^{s}, \quad r>0 \\
u(0)=a>0 \\
u^{\prime}(0)=0
\end{array}\right.
$$

с $u^{\prime}(r) \neq 0$ при $r>0$.

В случае $p=2$, когда $\Delta_{p}$ совпадает с обычньм оператором Лапласа, уравнение (1.1) было, насколько нам известно, впервые рассмотрено в пионерской работе Шипо и Вайслера [3] как стационарная версия параболического уравнения

$$
u_{t}-\Delta u=\lambda u^{q}-|\nabla u|^{s}, \quad x \in \Omega \subset \mathbb{R}^{n}, \quad t \in \mathbb{R} .
$$

Их техника была основана на методе стрельбы и изменении масштаба. В настоящей работе мы показываем, что эти методы напрямую обобщаются на случай задачи (1.1) 
c $1<p \leqslant 2$. Имеются, по крайней мере, два показателя, которые разграничивают различные типы результатов: показатель Соболева $q^{*}=(n+2) /(n-2)$ (который переходит в $\infty$ при $n=1,2)$ и показатель самоподобия $s=2 q /(q+1)$. Хорошо известно, что показатель Соболева играет важную роль при исследовании уравнения Лане-Эмдена

$$
-\Delta u=u^{q}
$$

и что, в частности, решения задачи Дирихле для этого уравнения в ограниченных областях существуют в точности при $1<q<q^{*}$. С другой стороны, для обобщенной версии этого уравнения

$$
-\Delta_{p} u=u^{q}
$$

аналогичньй результат имеет место с $q^{*}=n p /(n-p)-1$. Это мотивирует обобщение результатов [3] на (1.1) при $1<q<q^{*}$. В этом случае показатель самоподобия равен $s_{*}=p q /(q+1)$. Оставшаяся часть статьи имеет следующую структуру. В п. 2 мы получаем предварительные результаты о локальном существовании положительных решений (1.2) и их нулях. Вытекающие из них глобальные теоремы существования в случаях $p-1<s<s_{*}, s=s_{*}$ и $s>s_{*}$ доказьваются соответственно в пाп. 3-5.

2. Предварительные результаты. В дальнейшем на протяжении всей статьи будем подразумевать, что $1<p \leqslant 2, q \geqslant 1, s \geqslant p-1$. Как будет показано в следующей лемме, эти условия гарантируют локальную разрешимость задачи (1.2).

Лемма 2.1. Зафиксируем $\lambda>0$. Для любого а $>0$ существует (единственное) максимальное решение $u \in C^{2}\left(\left[0, R_{a}\right)\right)$ задачи (1.2). Более того, оно обладает следующими свойствами:

1) $u^{\prime}(r)<0$ для всех $r, 0<r<R_{a}$;

2) функиия

$$
H(r)=\frac{p-1}{p}\left|u^{\prime}(r)\right|^{p}+\frac{\lambda}{q+1}|u(r)|^{q} u(r)
$$

убивает на интервале $\left[0, R_{a}\right)$;

3) если $u(r)>0$ для всех $r, 0 \leqslant r<R_{a}$, mо $R_{a}=\infty u$

$$
\lim _{r \rightarrow \infty} u(r)=\lim _{r \rightarrow \infty} u^{\prime}(r)=\lim _{r \rightarrow \infty} u^{\prime \prime}(r)=0 .
$$

ДокАЗАтЕльство. Докажем сначала существование единственного решения задачи (1.2) на некотором интервале $[0, \varepsilon]$. Умножая уравнение в $(1.2)$ на $r^{n-1}$ и интегрируя его с учетом начальных условий, получаем, что любое решение этой задачи удовлетворяет системе интегральных уравнений

$$
\left\{\begin{array}{l}
u(r)=a+\int_{0}^{r}|w(\rho)|^{(2-p) /(p-1)} w(\rho) d \rho \\
w(r)=r^{1-n} \int_{0}^{r} \rho^{n-1}\left(|w(\rho)|^{s /(p-1)}-\lambda|u(\rho)|^{q}\right) d \rho
\end{array}\right.
$$

c $w(r)=\left|u^{\prime}(r)\right|^{p-2} u^{\prime}(r)$. Применяя принцип сжимающих отображений, получаем единственное решение системы $(2.1) u, w \in C([0, \varepsilon])$ для некоторого $\varepsilon>0$. Очевидно, $u \in$ 
$C^{1}([0, \varepsilon])$ с $u^{\prime}(r)=|w(r)|^{(2-p) /(p-1)} w(r)$. В частности, $u^{\prime}(0)=0$. Более того, $w$ принадлежит $C^{1}((0, \varepsilon])$, и поэтому $u \in C^{2}((0, \varepsilon])$ и удовлетворяет $(1.2)$. Остается показать, что при $r=0$ решение $u$ лежит в $C^{2}$. Для этого покажем сначала, что функция $w$ непрерьвно дифференцируема в нуле. Из (2.1) легко получить

$$
\begin{aligned}
w^{\prime}(0) & =\lim _{r \rightarrow 0} \frac{w(r)}{r}=\lim _{r \rightarrow 0} \frac{1}{r^{n}} \int_{0}^{r} \rho^{n-1}\left(|w(\rho)|^{s /(p-1)}-\lambda|u(\rho)|^{q}\right) d \rho \\
& =\lim _{r \rightarrow 0} \frac{r^{n-1}\left(|w(r)|^{s /(p-1)}-\lambda|u(r)|^{q}\right)}{n r^{n-1}}=-\frac{\lambda a^{q}}{n} .
\end{aligned}
$$

С другой стороны, из (1.2) вытекает

$$
\begin{aligned}
\lim _{r \rightarrow 0} w^{\prime}(r) & =-\lim _{r \rightarrow 0}\left(\frac{(n-1) w(r)}{r}+\lambda u^{q}(r)-|w(r)|^{s /(p-1)}\right) \\
& =\frac{(n-1) \lambda a^{q}}{n}-\lambda a^{q}=-\frac{\lambda a^{q}}{n} .
\end{aligned}
$$

Таким образом, $w \in C^{1}([0, \varepsilon])$, и поэтому $u \in C^{2}([0, \varepsilon])$.

Чтобы доказать 1$)$, заметим сначала, что $u(0)=a>0$ и $w(0)=0$. В силу $(2.2)$ имеем $w^{\prime}(0)<0$, что в сочетании с $w(0)=0$ дает $w(r)<0$ на некотором интервале $(0, \delta]$. Обозначим через $r_{0}$ первьй положительный нуль функций $u^{\prime}$ и $w$. Тогда $u\left(r_{0}\right) \neq 0$, так как в случае $u\left(r_{0}\right)=w\left(r_{0}\right)=0$ из теоремы единственности для системы (2.1) вытекает, что $u(r) \equiv 0$. Следовательно, из уравнения в (1.2) имеем

$$
w^{\prime}\left(r_{0}\right)=-\lambda\left|u\left(r_{0}\right)\right|^{q}<0,
$$

откуда $w(r)$ и соответственно $u^{\prime}(r)$ положительны для всех $r$ в некотором интервале $\left(r_{0}-\eta, r_{0}\right)$ с $\eta>0$. Это противоречит выбору $r_{0}$ и поэтому доказывает 1$)$.

Так как при $r>0$ уравнение (1.2) не содержит особенностей, решение на $[0, \varepsilon]$ можно локально продолжить до максимального $u \in C^{2}\left(\left[0, R_{a}\right]\right)$. Вспомним, что в процедуре продолжения (1.2) рассматривается как система относительно $(u(r), w(r))$ с $w(r)=$ $\left|u^{\prime}(r)\right|^{p-2} u^{\prime}(r)$. Следовательно, если этот процесс останавливается при некотором $R_{a}<\infty$, это означает, что либо $|u(r)| \rightarrow \infty$, либо $\left|u^{\prime}(r)\right| \rightarrow \infty$ при $r \rightarrow R_{a}$. Очевидно, доказательство 1$)$ применимо ко всему интервалу $\left[0, R_{a}\right]$.

Далее легко вычислить, что при $r>0$

$$
\begin{aligned}
H^{\prime}(r) & =(p-1) u^{\prime}(r)\left|u^{\prime}(r)\right|^{p-2} u^{\prime \prime}(r)+\lambda|u(r)|^{q} u^{\prime}(r) \\
& =u^{\prime}(r)\left[-\left(\frac{n-1}{r}\right)\left|u^{\prime}(r)\right|^{p-2} u^{\prime}(r)+\left|u^{\prime}(r)\right|^{s}\right]<0,
\end{aligned}
$$

и поэтому выполнено 2).

Остается доказать 3$)$. Если $u(r)>0$ для всех $r \in\left[0, R_{a}\right)$, то $0<H(r) \leqslant H(0)$ для всех $r \in\left[0, R_{a}\right]$. Таким образом, $u(r)$ и $u^{\prime}(r)$ априори ограничены, так что $R_{a}=\infty$. Кроме того, по доказанному, $u^{\prime}(r)<0$. Поэтому существует конечньй предел

$$
\lim _{r \rightarrow \infty} u(r)=u_{\infty} .
$$


Аналогично, $H(r)$ стремится к конечному пределу при $r \rightarrow \infty$. Это влечет существование

$$
\lim _{r \rightarrow \infty} u^{\prime}(r)=v_{\infty}
$$

Фактически из сушествования $\lim _{r \rightarrow \infty} u(r)$ вытекает, что $v_{\infty}=0$, а поэтому и $w_{\infty}=$ $-\left|v_{\infty}\right|^{p-1}=0$. Наконец, из (1.2) мы заключаем, что

$$
\lim _{r \rightarrow \infty} w^{\prime}(r)=-\lambda\left|u_{\infty}\right|^{q} .
$$

Таким образом, $v_{\infty}=0$ можно получить только при $u_{\infty}=0$. Это завершает доказательство 3).

При фиксированном $\lambda>0$ обозначим первый нуль решения (1.2) через $z(a)$. Будем считать, что $z(a)=\infty$, если $u(r)>0$ для всех $r \geqslant 0$. Таким образом, решение $u(r)$ задачи (1.2) удовлетворяет (1.1) в точности при $z(a)=R$. Это побуждает исследовать поведение функции $z(a)$.

ЛЕмма 2.2. При условиях леммы 2.1 множсество $\{a>0: z(a)<\infty\}$ открыто, и функиия $z(\cdot)$ непрерывна на нем. Более того, если $q>p-1$, то

$$
\lim _{a \rightarrow 0} z(a)=\infty
$$

а если, кроме того, $z\left(a_{0}\right)=\infty$ при некотором $a_{0}>0$, mо $\lim _{a \rightarrow a_{0}} z(a)=\infty$.

ДокАЗАТЕЛЬСТво. Обозначим решение $u(r)$, полученное, как описано в предыдущем доказательстве, для любого фиксированного $a$ как $u(r, a)$. Если $z\left(a_{0}\right)<\infty$, то по его определению существуют числа $\delta<0$ и $\varepsilon>0$ такие, что $u\left(r, a_{0}\right)<\delta$ для всех $r \in\left[z\left(a_{0}\right)+\varepsilon, z\left(a_{0}\right)+2 \varepsilon\right]$. По теореме о непрерьвной зависимости $u$ от начальных данных, для всех $a$, достаточно близких к $a_{0}$, на том же интервале должно выполняться неравенство $u(r, a)<\delta / 2$. С другой стороны, $u(0, a)=a>0$. Поэтому $u$ должно обращаться в нуль в некоторой точке между 0 и $z\left(a_{0}\right)+\varepsilon$. Непрерьвность $z$ следует из непрерьвной зависимости $u(r, a)$ от $a$ и того, что $u$ может иметь не более одного нуля, так как $u^{\prime}(r)<0$ при $r>0$.

Чтобы доказать $(2.4)$, заметим, что так как $H(r)$ убьвает, то при всех $r \in\left[0, z_{a}\right]$

$$
\frac{p-1}{p}\left|u^{\prime}(r, a)\right|^{p} \leqslant H(r) \leqslant H(0)=\frac{\lambda a^{q+1}}{q+1}
$$

и поэтому

$$
\left|u^{\prime}(r, a)\right| \leqslant\left(\frac{p \lambda}{(p-1)(q+1)}\right)^{1 / p} a^{(q+1) / p}
$$

Следовательно,

$$
a=u(0, a)-u(z(a), a)=-\int_{0}^{z(a)} u^{\prime}(\rho, a) d \rho \leqslant z(a) a^{(q+1) / p}\left(\frac{p \lambda}{(p-1)(q+1)}\right)^{1 / p}
$$

т.e.

$$
z(a) \geqslant a^{-(q+1-p) / p}\left(\frac{(p-1)(q+1)}{p \lambda}\right)^{1 / p}
$$


и доказательство (2.4) завершено.

Чтобы доказать последнее утверждение, покажем, что при данном $M>0$ выполняется неравенство $z(a)>M$, если $a$ достаточно близко к $a_{0}$. Действительно, при $a=a_{0}$ имеем $u\left(r, a_{0}\right)>0$ для всех $r>0$; поэтому существует $\delta>0$ такое, что $u\left(r, a_{0}\right) \geqslant \delta$ на $[0, M]$. В силу непрерывной зависимости решения системы $(2.1)$ от начальных данных, если $a$ достаточно близко к $a_{0}$, то $u(r, a) \geqslant \delta / 2$ на $[0, M]$. Поэтому $z(a)>M$ для таких $a$.

3. Первый случай: $p-1 \leqslant s<p q /(q+1)$. Далее рассмотрим поведение функции $z(a)$ при $a \rightarrow \infty$. Начнем со случая $s<p q /(q+1)$.

ЛЕмма 3.1. Пусть $q>1, \quad p-1 \leqslant s<p q /(q+1) u($ в случае $n>p) 1<q<$ $n p /(n-p)-1$. Тогда для всех $\lambda>0$ имеем

$$
\lim \sup _{a \rightarrow \infty} z(a) a^{(q-p+1) / p}<\infty
$$

u поэтому

$$
\lim _{a \rightarrow \infty} z(a)=0
$$

ДокАзАТЕЛьство. Зафиксируем $\lambda>0$. Как в предыдущем параграфе, обозначим через $u(\cdot, a)$ решение задачи $(1.2)$ с начальным значением $a$. Для всех $a>0$ положим

$$
v_{a}(r)=a^{-1} u\left(r a^{(p-q-1) / p}, a\right) .
$$

Тогда легко видеть, что $v_{a}$ удовлетворяет соотношениям

$$
\left\{\begin{array}{l}
-\left((p-1) v_{a}^{\prime \prime}(r)+\frac{n-1}{r} v_{a}^{\prime}(r)\right)\left|v_{a}^{\prime}(r)\right|^{p-2} \\
\quad=\lambda\left|v_{a}(r)\right|^{q}-a^{(s(q+1)-p q) / p}\left|v_{a}^{\prime}(r)\right|^{s}, \quad r>0 \\
v_{a}(0)=1 \\
v_{a}^{\prime}(0)=0
\end{array}\right.
$$

Кроме того, $v_{a}^{\prime}(r)<0$ при $r>0$ и $v_{a}(r)>0$ при $0 \leqslant r<a^{(q-p+1) / p} z(a)$, и поэтому

$$
0 \leqslant v_{a}(r) \leqslant 1, \quad 0 \leqslant r \leqslant a^{(q-p+1) / p} z(a)
$$

Более того, (2.5) переходит в

$$
\left|v_{a}^{\prime}(r)\right| \leqslant\left(\frac{p \lambda}{(p-1)(q+1)}\right)^{1 / p}, \quad r \geqslant 0
$$

$\mathrm{a}(2.2)-\mathrm{B}$

$$
\lim _{r \rightarrow 0} \frac{\left|v_{a}^{\prime}(r)\right|^{p-2} v_{a}^{\prime}(r)}{r}=-\frac{\lambda}{n} .
$$

Из (3.3)-(3.6) вытекает также равномерная по $r$ и $a$ при $0 \leqslant r<a^{(q-p+1) / p} z(a)$ и $a \geqslant a_{0}>0$ оценка для $\left|v_{a}^{\prime \prime}(r)\right|$. 
Теперь предположим, что сушествует последовательность $a_{m} \rightarrow \infty$ такая, что выполняется

$$
a_{m}^{(q-p+1) / p} z\left(a_{m}\right) \rightarrow \infty \quad \text { при } m \rightarrow \infty .
$$

Используя теорему Арцела-Асколи и стандартный диагональньй процесс, получим подпоследовательность, которую снова обозначим $a_{m}$, и непрерьвную функцию $v:[0, \infty) \rightarrow$ $[0,1]$ такую, что $v_{a_{m}} \rightarrow v$ и $v_{a_{m}}^{\prime} \rightarrow v^{\prime}$ равномерно на всех компактных подмножествах $[0, M] \subset[0, \infty)$. В частности, $v(0)=1, v$ неотрицательно, не возрастает на $[0, \infty)$ и непрерьвно по Липшицу с константой, не превосходящей $(p \lambda /((p-1)(q+1)))^{1 / p}$. (Каждая из функций $v_{a}$ имеет эти свойства.) Наконец, так как $s<p q /(q+1)$ и $\left|v_{a}^{\prime}\right|,\left|v_{a}^{\prime \prime}\right|$ ограничены независимо от $r$ и $a$, из (3.3) следует, что

$$
\left((p-1) v^{\prime \prime}(r)+\frac{n-1}{r} v^{\prime}(r)\right)\left|v^{\prime}(r)\right|^{p-2}+\lambda|v(r)|^{q}=0
$$

в смысле распределений на $(0, \infty)$.

Хорошо известно (см., например, [4]), что при $1<q<n p /(n-p)-1$ такое регулярное решение $v$ не может существовать. Это доказьвает (3.1), а следовательно, и (3.2).

Tеорема 3.1. Пусть $1<p \leqslant 2, q>1, p-1 \leqslant s<p q /(q+1), \lambda>0 u($ ecлu $n>p)$ $q<n p /(n-p)-1$. Тогда задача (1.1) имеет решение $\varphi \in C^{2}\left(B_{R}\right)$.

ДокАЗАТЕЛЬство. Вследствие лемм 2.2 и 3.1 для любых $\lambda>0$ и $R>0$ существует $a>0$ такое, что $z(a)=R$. Иньми словами, если $u(r)-$ решение $(1.2)$ с начальньм значением $a$, то $u(r)>0$ при $0 \leqslant r<R$ и $u(R)=0$. Тогда $\varphi(x)=u(|x|),|x| \leqslant R-$ искомое решение задачи (1.1).

4. Второй случай: $s=p q /(q+1)$. Перейдем к случаю $s=p q /(q+1)$. Он отличается от предыдушего тем, что масштабированное решение $v_{a}$ удовлетворяет тому же уравнению, что и $и$. Это усложняет рассмотрение, поэтому мы ограничимся подслучаем $n=1$.

ЛЕмма 4.1. Если $s=p q /(q+1)$, то для всех $a>0$

$$
z(a)=a^{-(q-p+1) / p} z(1) .
$$

ДокАЗАТЕЛьСтво. В силу $(3.3) v_{a}(\cdot)=u(\cdot, 1)$ для всех $a>0$. Поэтому $v_{a}$ впервые достигает нуля в точке $z(1)$. Однако по определению $v_{a}$ его первым нулем является $a^{(q-p+1) / p} z(a)$. Это доказьвает (4.1).

Иными словами, конечно $z(a)$ или нет - зависит только от конечности $z(1)$, которая, в свою очередь, определяется значением параметра $\lambda$.

ЛЕМма 4.2. Пусть $r_{0} \geqslant 0, \lambda>0, s=p q /(q+1)$, и предположсим, что функиия $u:\left(r_{0}, \infty\right) \rightarrow \mathbb{R}$ принадлежстт $C^{2}$ и для всех $r, r>r_{0}$, удовлетворяет условиям

1) $u(r)>0 u \lim _{r \rightarrow \infty} u(r)=0$;

2) $u^{\prime}(r)<0 u \lim _{r \rightarrow \infty} u^{\prime}(r)=0$;

3) $(p-1) u^{\prime \prime}(r)\left|u^{\prime}(r)\right|^{p-2}-\left|u^{\prime}(r)\right|^{s}+\lambda|u(r)|^{q}=0$;

4) выполнена оценка

$$
u^{\prime \prime}(r) \leqslant k\left|u^{\prime}(r)\right|^{s-p+2},
$$

әде $k>0$ - фиксированная константа. 
Тогда для всех $r, r>r_{0}$, функиия и удовлетворяет такжсе неравенству

$$
u^{\prime \prime}(r) \leqslant \frac{1}{p-1}\left[1-\lambda\left(\frac{q+1}{p k}\right)^{q}\right]\left|u^{\prime}(r)\right|^{s-p+2} .
$$

ДокАЗАТЕЛьСтво. Так как $u^{\prime}<0$, неравенство (4.2) можно переписать в виде

$$
\left(-u^{\prime}(\rho)\right)^{p-s-1} u^{\prime \prime}(\rho) \leqslant-k u^{\prime}(\rho) .
$$

Интегрируя его от $r$ до $\infty$, получим

$$
\frac{\left(-u^{\prime}(r)\right)^{p-s}}{p-s} \leqslant k u(r)
$$

или, что то же самое,

$$
u(r) \geqslant \frac{q+1}{p k}\left(-u^{\prime}(r)\right)^{p /(q+1)}
$$

(здесь мы используем, что $s=p q /(q+1)<p$ ). Поэтому в силу условия 3 )

$$
(p-1) u^{\prime \prime}(r)\left|u^{\prime}(r)\right|^{p-2}=\left(-u^{\prime}(r)\right)^{s}-\lambda u^{q}(r) \leqslant\left[1-\lambda\left(\frac{q+1}{p k}\right)^{q}\right]\left(-u^{\prime}(r)\right)^{s},
$$

что доказьвает (4.3).

ЛЕмма 4.3. Пусть $n=1 u s=p q /(q+1)$. Ecлu $\lambda \geqslant p^{q} /((p-1)(q+1))^{q}, m o$ $z(a)<\infty$ для всех решений $и(r)$ задачи (1.2).

ДоКАЗАТЕЛЬСТво. Если $z(a)=\infty$, то $u(r)>0$ для всех $r>0$. В силу леммы 2.1 и уравнения (1.2) с $n=1$ функция $u(r)$ удовлетворяет условиям 1)-3) леммы 4.2, а также условию 4$)$ с $k=1 /(p-1)$. Поэтому из леммы 4.2 следует, что (4.3) вьполняется с тем же $k$. Так как $\lambda \geqslant p^{q} /((p-1)(q+1))^{q}$, имеем $u^{\prime \prime}(r) \leqslant 0$ для $r>0$. Но это невозможно, так как $u^{\prime}(r)<0$ и $u(r)>0$ при $r>0$.

Результат леммы 4.3 уже достаточен, чтобы получить решение (1.1) с

$$
\lambda \geqslant\left(\frac{p}{(p-1)(q+1)}\right)^{q} .
$$

Тем не менее, его можно усилить.

ЛЕмма 4.4. Пусть $\lambda>\lambda_{p, q}=(p q)^{q} /\left((p-1)^{q}(q+1)^{2 q+1}\right)$. Индуктивно определим последовательность

$$
k_{0}=\frac{1}{p-1}, \quad k_{m}=\frac{1}{p-1}\left(1-\lambda\left(\frac{q+1}{p k_{m-1}}\right)^{q}\right)
$$

при $k_{m-1}>0$. Тогда либо $k_{m}$ при некотором $m$ станет неположстельным, либо $\lim _{m \rightarrow \infty} k_{m}=0$. 
ДокАЗАТЕЛЬСТво. Рассмотрим функцию

$$
f_{\lambda}(x)=x-\frac{1}{p-1}\left(1-\lambda\left(\frac{q+1}{p x}\right)^{q}\right)
$$

которая, очевидно, задает значения разности $k_{m-1}-k_{m}$ при $x=k_{m-1}$. Для $\lambda>\lambda_{p, q}$ и $x>0$ имеем

$$
f_{\lambda}(x)>f_{\lambda_{p, q}}(x)=x-\frac{1}{p-1}\left(1-\frac{1}{q+1}\left(\frac{q}{(p-1)(q+1) x}\right)^{q}\right) .
$$

Легко проверить, что функция $f_{\lambda_{p, q}}$ достигает своего единственного минимума при $x_{0}=$ $q /((p-1)(q+1))$, причем $f_{\lambda_{p, q}}\left(x_{0}\right)=0$. Поэтому $f_{\lambda}(x)>0$ при $\lambda>\lambda_{p, q}$ и $x>0$, и последовательность $k_{m}$ убьвает, пока она определена. Следовательно, если заключение леммы неверно, то существует $\lim _{m \rightarrow \infty} k_{m}=k>0$ и в силу $(4.4) f_{\lambda}(k)=0$, что противоречит предыдущему рассуждению. Лемма доказана.

ЛЕмма 4.5. Пусть $n=1$ us $=p q /(q+1)$. Если $\lambda>\lambda_{p, q}$, mo $z(a)<\infty$ для всех решений (1.2).

ДокАЗАТЕЛЬСТво. Повторяя рассуждения из доказательства леммы 4.3 и применяя лемму 4.2 необходимое количество раз, получаем, что (4.3) вьполняется при всех значениях $k=k_{m}$, определенных в лемме 4.4. Следовательно, $u^{\prime \prime}(r)<0$ для всех $r>0$. Это приводит к противоречию аналогично доказательству леммы 4.3 .

Tеорема 4.1. Пусть $1<p \leqslant 2, q \geqslant 1, s=p q /(q+1), n=1 u \lambda>\lambda_{p, q}$. Тогда задача (1.1) имеет решение $u \in C^{2}\left(B_{R}\right)$.

ДокАЗАТЕЛЬСТво. Достаточно воспроизвести доказательство теоремы 3.1 со ссылкой на леммы 4.1 и 4.5 вместо леммы 3.1 .

5. Третий случай: $s>p q /(q+1)$. Нам остается рассмотреть случай $s>p q /(q+1)$. Следующий результат - вариант леммы 2.2 .

ЛЕмма 5.1. Пусть $\lambda>0 u и(r)$-решение (1.2), впервье обращающееся в нуль в точке $z(a)$. Тогда

$$
z(a) \geqslant \lambda^{-1 / s} a^{1-q / s} .
$$

ДокАЗАТЕЛьСтво. Предположим, что $z(a)<\infty$. Покажем, что минимальное значение функции $u^{\prime}(r)$ и, соответственно, $w(r)=u^{\prime}(r)\left|u^{\prime}(r)\right|^{p-2}$ на $[0, z(a)]$ достигается внутри интервала. Действительно, так как $u^{\prime}(r)<0$, имеем

$$
w^{\prime}(r)=\left(-\left|u^{\prime}(r)\right|^{p-1}\right)^{\prime}=(p-1) u^{\prime \prime}(r)\left|u^{\prime}(r)\right|^{p-2}
$$

Но в силу (1.2) и (2.3)

$$
\begin{aligned}
(p-1) u^{\prime \prime}(0)\left|u^{\prime}(0)\right|^{p-2} & =-\frac{\lambda a^{q}}{n}<0, \\
(p-1) u^{\prime \prime}(z(a))\left|u^{\prime}(z(a))\right|^{p-2} & =-\left(\frac{n-1}{z(a)}\right) u^{\prime}(z(a))\left|u^{\prime}(z(a))\right|^{p-2}+\left|u^{\prime}(z(a))\right|^{s}>0 .
\end{aligned}
$$


Поэтому, если $r_{0}$ таково, что $w\left(r_{0}\right)$ - минимум на $[0, z(a)]$, то $w^{\prime}\left(r_{0}\right)=0$, т.е.

$$
-\left(-u^{\prime}\left(r_{0}\right)\right)^{s}+\lambda\left(u\left(r_{0}\right)\right)^{q}=-\frac{n-1}{r_{0}} u^{\prime}\left(r_{0}\right)\left|u^{\prime}\left(r_{0}\right)\right|^{p-2} \geqslant 0
$$

или

$$
\left(-u^{\prime}\left(r_{0}\right)\right)^{s} \leqslant \lambda\left(u\left(r_{0}\right)\right)^{q} \leqslant \lambda a^{q} .
$$

Отсюда следует, что для $0 \leqslant r \leqslant z(a)$ вьполнено $-u^{\prime}(r) \leqslant \lambda^{1 / s} a^{q / s}$. Следовательно,

$$
a=u(0)-u(z(a))=-\int_{0}^{z(a)} u^{\prime}(\rho) d \rho \leqslant z(a) \lambda^{1 / s} a^{q / s},
$$

что доказьвает (5.1).

Лемма 5.2. Пусть $q>1, s>p q /(q+1) u($ в случае $n>p) q<n p /(n-p)-1$. Тогда для всех $\lambda>0$ имеем

$$
\lim \sup _{a \rightarrow 0} z(a) a^{(q-p+1) / p}<\infty .
$$

В частности, для всех достаточно малых а $z(a)<\infty u$

$$
z(a) \leqslant C a^{-(q-p+1) / p}
$$

с некоторым $C>0$.

ДоКАЗАТЕЛЬСТВо есть очевидная модификация доказательства леммы 3.1.

Обозначим для любого фиксированного $\lambda>0$

$$
R(\lambda)=\inf _{a>0} z(a)
$$

Заметим, что вследствие леммы 2.2 при $R(\lambda)<\infty$ уравнение $z(a)=R$ имеет, по крайней мере, одно решение для любого $R>R(\lambda)$.

Лемма 5.3. Пусть $q>1$. Тогда

1) $е$ eсли $s \geqslant q$, mo $R(\lambda)>0$;

2) если $s>p q /(q+1) u($ в случае $n>p) \quad q<n p /(n-p)-1$, mo $R(\lambda)<\infty$;

3) если $s>q u($ в случае $n>p) q<n p /(n-p)-1$, mо $z(a)=R(\lambda)<\infty$ для некоторого а $>0$, и уравнение $z(a)=R$ имеет, по крайней мере, два решения $a>0$ для любого $R>R(\lambda)$.

ДокАЗАТЕЛьСТво. Утверждение 1) следует из оценок снизу для $z(a)(2.6)$ и (5.1), а утверждение 2) - из леммы 5.2. Чтобы доказать утверждение 3), заметим, что из (2.6) и (5.1) следует

$$
\lim _{a \rightarrow 0} z(a)=\lim _{a \rightarrow \infty} z(a)=\infty .
$$

Но в силу леммы $5.2 z(a)$ конечно для некоторых $a$ и с учетом леммы 2.2 достигает положительного минимума $R(\lambda)$ при некотором $a_{0}, 0<a_{0}<\infty$. Очевидно, для любого $R>R(\lambda)$ сушествуют $a_{1}$ и $a_{2}$ такие, что $0<a_{1}<a_{0}<a_{2}<\infty$ и $z\left(a_{1}\right)=z\left(a_{2}\right)=R$.

Аналогично предыдущим пунктам, из леммы 5.3 следует 
Teоpema 5.1. Пусть $1<p \leqslant 2, q>1 u s \geqslant p-1$. Тогда

1) если $s \geqslant q u 0<R<R(\lambda)$, задача (1.1) не имеет (классических) радиальных решений в $B_{R}(0)$

2) если $s>p q /(q+1)$ u (в случае $n>p) \quad q<n p /(n-p)-1$, то при $R>R(\lambda)$ задача (1.1) имеет, по крайней мере, одно радиальное решение в $B_{R}(0)$;

3) если $s>q$ и (в случае $n>p) \quad q<n p /(n-p)-1$, то при $R=R(\lambda)$ задача (1.1) имеет, по крайней мере, одно, а при $R>R(\lambda)$ - по крайней мере, два радиальных решения в $B_{R}(0)$.

Автор благодарит С. И. Похожаева и К. О. Бесова за ценную помощь при подготовке рукописи к печати.

\section{СПИСОК ЦИТИРОВАННОЙ ЛИТЕРАТУРЫ}

[1] Gidas B., Ni W.-M., Nirenberg L. Symmetry and related properties via the maximum principle // Comm. Math. Phys. 1979. V. 68. P. 209-243.

[2] Mitidieri E., Caristi G. Nonexistence of positive solutions of quasilinear equations // Adv. Differential Equations. 1997. V. 2. P. 319-359.

[3] Chipot M., Weissler F. B. Some blow up results for a nonlinear parabolic problem with a gradient term // SIAM J. Math. Anal. 1989. V. 20. P. 886-907.

[4] Ni W.-M., Serrin J. Existence and non-existence theorems for ground states of quasilinear partial differential equations. The anomalous case // Accad. Naz. Lincei, Convegni dei Lincei. 1986. V. 77. P. 231-256. 